\title{
Deception Jamming Against Doppler Beam Sharpening Radars
}

\author{
Gareth Frazer and Alessio Balleri \\ Centre for Electronic Warfare, Information and Cyber (EWIC) \\ Cranfield University \\ Defence Academy of the United Kingdom \\ Shrivenham, SN6 8LA \\ Email: g.j.frazer@cranfield.ac.uk
}

\author{
George S. Jacob \\ The Defence Science and Technology Laboratory \\ United Kingdom
}

\begin{abstract}
Missile seekers are becoming increasingly more capable of using Doppler Beam Sharpening (DBS) modes as part of the homing cycle. This paper develops jamming theory and uses it for practical implementation of inserting false targets into a DBS image. The theory is also developed for how incorrect estimations of the seeker trajectory can affect the quality and location of the false target. The simulated and experimental results show how received missile seeker waveforms can be modified to create false targets at desired locations.
\end{abstract}

\section{INTRODUCTION}

Doppler Beam Sharpening (DBS) is a technique that uses the changing Doppler caused by relative motion between a platform and a target, to synthetically narrow the main beam of the radar. Due to the constraints of the missile, seeker antennas are relatively small and therefore have wider beams and larger cross-range resolutions than desired. Missile seekers benefit from using DBS, as it improves cross-range resolution without being computationally expensive like Synthetic Aperture Radars (SAR) and DBS images are generally much faster to obtain than SAR. As a generic example, DBS can be performed with a dwell time in the order of tens of milliseconds, whereas SAR is in the order of whole seconds [1].

The use of DBS for missile homing gives rise to the requirement for countermeasures against this mode. In order for a jammer to create a false target at a desired location within the seeker DBS image and with the correct focus, the jammer must create a synthetic phase history to mimic the phase history a target would have if it was at that location. This requires knowledge of the missile trajectory as well as the ability to coherently modify the seeker waveform. The jammer also needs to detect that the seeker is performing DBS and not using another mode for identifying targets. Detecting when a seeker would use DBS is beyond the scope of this paper, but the work set out in [2], [3] and [4], suggests that there is an optimal and specific trajectory that the missile could use to perform DBS. Prior knowledge of these types of trajectories could enable the jammer to detect that the seeker is using the DBS mode.

The ability to create high fidelity copies of waveforms and modify them comes from the use of Digital Radio Frequency
Memory (DRFM) systems. A DRFM can store and modify waveforms using high-speed sampling and digital memory [5]. The DRFM enables phase coherency which enables coherent radar modes to be jammed such as by creating false targets in SAR images [6]. There is very little literature on jamming DBS modes, but previous work in [7] introduced the concept of creating false targets in DBS images of a missile seeker. However, it did not explain how errors are induced by incorrect position and velocity estimations, nor did it discuss the practical implementations of waveform modification.

\section{DBS THEORY AND SCENARIO}

The achievable range and cross-range resolution of a DBS radar is essentially determined by the bandwidth of the transmitted waveform and the dwell time of the Coherent Processing Interval (CPI). Pulse compression waveforms such as Linear Frequency Modulation (LFM) or Stepped Frequency increase the effective bandwidth of the pulse and increase range resolution capabilities. The theoretical Doppler resolution of a DBS radar is the inverse of the dwell time $\left(\delta f_{d}=\frac{1}{T_{d}}\right)$. Azimuth "compression" is achieved by taking the Fourier transform across each range bin.

The range profiles of the targets can be found by the crosscorrelation function between the received signal $s_{r}(t)$ and the transmitted pulse, $s_{t}(t)$ as

$$
s_{M F}(t)=s_{t}(t) * s_{r}(t)=\int_{\infty}^{\infty} s_{t}^{*}(\tau-t) s_{r}(\tau) d \tau
$$

The Doppler history of the targets can then be found by taking the Fourier Transform across the pulses for each range bin $t_{0}$ as

$$
S\left(t_{0}, f\right)=\sum_{m=1}^{M-1} s_{M F}\left(t_{0}+m P R I\right) e^{-i 2 \pi f m P R I}
$$

where PRI is the Pulse Repetition Interval. If the trajectory of the missile is linear during one CPI of the radar and has no acceleration in any direction, the velocity vector of the missile in $3 \mathrm{D}$ will be

$$
\vec{v}=V[\cos (\gamma) \cos (\chi) \hat{i}+\cos (\gamma) \sin (\chi) \hat{j}+\sin (\gamma) \hat{k}]
$$

Where $V$ is the missile speed and $\gamma$ and $\chi$ are the missile headings in elevation and azimuth, respectively. Assuming 
$r(\overrightarrow{0})=0$, this means the position vector of the missile as a function of time would be

$$
\begin{aligned}
r \overrightarrow{(t)} & =\int \vec{v} d t \\
& =V t[\cos (\gamma) \cos (\chi) \hat{i}+\cos (\gamma) \sin (\chi) \hat{j}+\sin (\gamma) \hat{k}]
\end{aligned}
$$

For simplicity and ease of presentation, we consider the 2D scenario for the remainder of the paper. We also assume a perpendicular trajectory and no range migration in the images where both $\gamma$ and $\chi=0^{\circ}$.

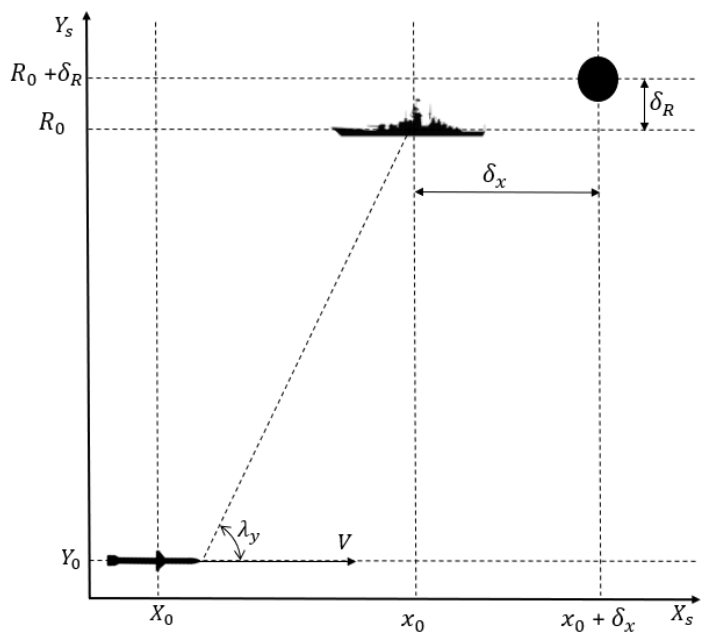

Fig. 1. Missile Reference Frame

From Figure 1, the position vector of a stationary target (the ship) $\vec{T}$ is given by

$$
\vec{T}=x_{0} \hat{i}+R_{0} \hat{j}
$$

where $x_{0}$ and $R_{0}$ are the cross-range and downrange positions from the missile. If the missile trajectory is such that it is moving closer to the target, the resultant range to go vector, $\overrightarrow{r_{T}}(t)$, is therefore

$$
\overrightarrow{r_{T}}(t)=\vec{T}-\vec{r}(t)=\left(x_{0}-V t\right) \hat{i}+R_{0} \hat{j}
$$

If the range between the seeker and the target is

$$
\left\|\overrightarrow{r_{T}}(t)\right\|=\sqrt{\left(x_{0}-V t\right)^{2}+R_{0}^{2}}=R_{T}(t)
$$

then the time delay of the echo from the target is

$$
\tau(t)=\frac{2\left\|\overrightarrow{r_{T}}(t)\right\|}{c}=\frac{2 \sqrt{\left(x_{0}-V t\right)^{2}+R_{0}^{2}}}{c}
$$

We study the quantity

$\sqrt{{R_{0}}^{2}+\left(x_{0}-V t\right)^{2}}=R_{0}\left(1+\frac{x_{0}^{2}}{{R_{0}}^{2}}-\frac{2 V t x_{0}}{R_{0}^{2}}+\frac{(V t)^{2}}{R_{0}^{2}}\right)^{\frac{1}{2}}$

When $R_{0}>>x_{0}, \frac{x_{0}{ }^{2}}{R_{0}{ }^{2}}$ and $\frac{(V t)^{2}}{R_{0}^{2}}$ can be approximated to zero. This means Eq. (9) can be simplified to

$$
R_{0}\left(1+\frac{x_{0}^{2}}{R_{0}^{2}}-\frac{2 V t x_{0}}{R_{0}^{2}}+\frac{(V t)^{2}}{R_{0}^{2}}\right)^{\frac{1}{2}} \simeq R_{0}\left(1-\frac{2 V t x_{0}}{R_{0}^{2}}\right)^{\frac{1}{2}}
$$

By taking the first order Taylor approximation of Eq. (10), the time delay in Eq. (8) can be expressed as

$$
\tau(t)=\frac{2 R_{0}}{c}-\frac{2 V x_{0} t}{c R_{0}}=\frac{2 R_{0}}{c}-\frac{2 \beta t}{c}
$$

with $\beta=\frac{V x_{0}}{R_{0}}$. An arbitrary waveform, transmitted and then received by the seeker from an echo reflected from a target is

$$
s_{R}(t)=x(t-\tau(t)) e^{i 2 \pi f_{c}(t-\tau(t))}
$$

where $f_{c}$ is the carrier frequency of the transmitted wave. The received signal after down-conversion will be

$$
s_{R}(t)=x\left(t-\frac{2 R_{0}}{c}\right) e^{-i 2 \pi f_{c}\left(\frac{2 R_{0}}{c}-\frac{2 \beta t}{c}\right)}
$$

Then the instantaneous frequency of that waveform is defined as the time derivative of the phase divided by $2 \pi$

$$
\begin{aligned}
f_{I F}= & \frac{1}{2 \pi} \frac{d\left[-2 \pi f_{c}\left(\frac{2 R_{0}}{c}-\frac{2 V t x_{0}}{c R_{0}}\right)\right]}{d t} \\
& =\frac{-\frac{2 f_{c}}{c} d\left[R_{0}-\frac{V t x_{0}}{R_{0}}\right]}{d t}
\end{aligned}
$$

This means that the Doppler shift for a target at the position in Eq. (5) would be

$$
f_{d}=\frac{2 V x_{0}}{\lambda R_{0}}
$$

The seeker measures the range and the Doppler shift and then inverts Eq. (16) to obtain the cross-range position $x_{0}$.

\section{JAMMING THEORY AND METHOD}

\section{A. Theory}

We now study the case of a self-protection jammer and for the scenario in Figure 1, the jammer is physically located on the ship. Instead of a target reflecting a received signal like in the previous section, this section includes an additional step. The jammer intercepts the transmitted seeker waveform and then both delays and modifies that waveform, following the scheme in Figure 2.

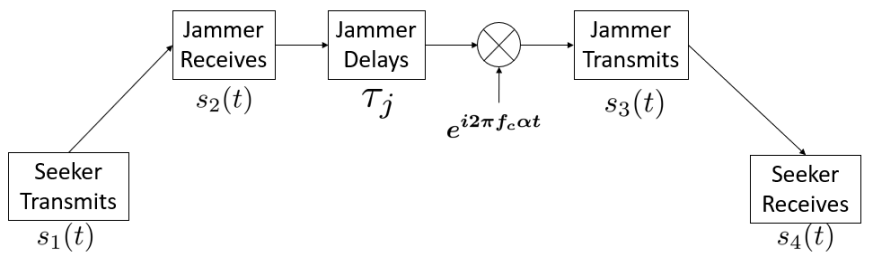

Fig. 2. Jamming Block Diagram

The block diagram in Figure 2 shows that the jammer would receive the signal $s_{2}(t)$ which is a delayed version of $s_{1}(t)$. 
The jammer would then delay $s_{2}(t)$ to give an additive range component and modify the waveform to change the Doppler profile of the target. Let $\tau_{j}$ be the additive jammer delay and $\alpha$ the modifying Doppler shift applied to the waveform. After downmixing, narrowband and previous approximations

$$
s_{4}(t)=x\left(t-\frac{2 R_{0}}{c}-\tau_{j}\right) e^{i 2 \pi f_{c} \frac{2 \beta}{c} t} e^{-i 2 \pi f_{c}\left[\frac{2 R_{0}}{c}+\tau_{j}\right]} e^{i 2 \pi f_{c} \alpha t}
$$

In order for the jammer to create the perfect false target at the range $R_{0}+\delta_{R}$ and cross-range $x_{0}+\delta_{x}$, the value of $\tau_{j}$ and $\alpha$ must be such that the result at the seeker would be

$$
s_{4}(t)=x\left(t-\frac{2\left(R_{0}+\delta_{R}\right)}{c}\right) e^{i 2 \pi f_{c} \frac{2 \beta_{J}}{c} t} e^{-i 2 \pi f_{c} \frac{2\left(R_{0}+\delta_{R}\right)}{c}}
$$

where

$$
\beta_{J}=\frac{V\left(x_{0}+\delta_{x}\right)}{\left(R_{0}+\delta_{R}\right)}
$$

The additional down and cross ranges added to make $\beta_{J}$ different to $\beta$, are used to show that an ideal jamming point target would look exactly like an echo received if a target was at the positions of $\left(x_{0}+\delta_{x}\right)$ and $\left(R_{0}+\delta_{R}\right)$ in the $\mathrm{x}$ and $\mathrm{y}$ axes respectively. This means that

$$
e^{i 2 \pi f_{c} \frac{2 \beta t}{c}} e^{i 2 \pi f_{c} \alpha t}=e^{i 2 \pi f_{c} \frac{2 \beta \beta_{J} t}{c}}
$$

and

$$
\alpha=\frac{2 \beta_{J}}{c}-\frac{2 \beta}{c}=\frac{2 V}{c}\left[\frac{\left(x_{0}+\delta_{x}\right)}{\left(R_{0}+\delta_{R}\right)}-\frac{x_{0}}{R_{0}}\right]
$$

This means that the signal received at the seeker would be

$$
\begin{aligned}
s_{4}(t) & =x\left(t-\frac{2 R_{0}}{c}-\tau_{j}\right) e^{i 2 \pi f_{c} \frac{2 \beta t}{c}} \\
& \times e^{-i 2 \pi f_{c}\left(\frac{2 R_{0}}{c}+\tau_{j}\right)} e^{i 2 \pi f_{c}\left(\frac{2 \beta J}{c}-\frac{2 \beta}{c}\right) t}
\end{aligned}
$$

Where $\tau_{j}=\frac{2 \delta_{R}}{c}$ and $\delta_{R}$ is the additive miss distance in downrange for the false target. Table I gives the seeker position variables and the additional false target positions for an example scenario.

TABLE I

EXAMPLE SCENARIO VARIABLES

\begin{tabular}{cccc}
\hline \hline Variable & Seeker & False Target & Miss Distance \\
\hline Down-range $(\mathrm{km})$ & 24 & 24.4 & 0.4 \\
Cross-range $(\mathrm{km})$ & 0.5 & 0.7 & 0.2 \\
\hline
\end{tabular}

The constant $\frac{2\left(\delta_{R}\right)}{c}$ can be considered to be the additive range component and the term $\frac{V t\left(x_{0}+\delta_{x}\right)}{\left(R_{0}+\delta_{R}\right)}-\frac{V t x_{0}}{R_{0}}$ is the phase component which will give the Doppler history and therefore determine the cross-range position of the false target. This approximation holds providing that there is no range migration during a CPI.
For a pulsed radar using Linear Frequency Modulated (LFM) waveforms, the transmitted pulse would be

$$
\begin{gathered}
x(t)=\sum_{m=0}^{M-1} s_{T}(t-m \mathrm{PRI}) e^{i 2 \pi f_{c} t} \\
s_{T}(t)=\operatorname{Rect}\left(\frac{t}{\tau}\right) e^{i \pi \gamma t^{2}} \\
\operatorname{Rect}\left(\frac{t}{\tau}\right)=\left\{\begin{array}{ll}
1, & 0<t<\tau \\
0, & \text { Elsewhere }
\end{array}\right\}
\end{gathered}
$$

This means that after down-conversion, the seeker would receive

$$
\begin{aligned}
s_{R}(t) & =\sum_{m=0}^{M-1} s_{T}\left(t-m \text { PRI }-\frac{2 R_{0}}{c}-\tau_{j}\right) e^{i 2 \pi f_{c} \frac{2 \beta t}{c}} \\
& \times e^{-i 2 \pi f_{c}\left(\frac{2 R_{0}}{c}+\tau_{j}\right)} e^{i 2 \pi f_{c} \alpha t}
\end{aligned}
$$

The time domain is now sampled in a fast-slow time form where $t=n T_{s}+k$ PRI with $n=0, . ., N-1$ where $N$ are the number of range bins $\left(N T_{s}=P R I\right)$ and $k=0, . ., M-1$. If $t_{0}=n T_{s}$, the received signal becomes

$$
\begin{aligned}
s_{R}\left(t_{0}+k \mathrm{PRI}\right) & =\sum_{m=0}^{M-1} s_{T}\left(t_{0}+(k-m) \mathrm{PRI}-\frac{2 R_{0}}{c}-\tau_{j}\right) \\
& \times e^{i 2 \pi f_{c} \frac{2 \beta}{c} t_{0}} e^{i 2 \pi f_{c} \frac{2 \beta}{c} k \mathrm{PRI}} e^{-i 2 \pi f_{c}\left(\frac{2 R_{0}}{c}+\tau_{j}\right)} \\
& \times e^{i 2 \pi f_{c} \alpha t_{0}} e^{i 2 \pi f_{c} \alpha k \mathrm{PRI}}
\end{aligned}
$$

Using the above equations, positions in Table I and the signal variables in Table II, Figure 3 demonstrates that a false target at a different cross-range position to the jammer can be induced into the seeker DBS image by incrementally applying a phase shift to the received seeker waveform.

TABLE II

SiMULATION WAVEFORM VARIABLES

\begin{tabular}{ccc}
\hline \hline Variable & Symbol & Value \\
\hline Carrier Frequency & $f_{c}$ & $36 \mathrm{GHz}$ \\
Bandwidth & $\mathrm{B}$ & $40 \mathrm{MHz}$ \\
Pulse Width & $\tau$ & $3 \mu s$ \\
Pulse Repetition Frequency & PRF & $10 \mathrm{kHz}$ \\
\hline
\end{tabular}

\section{B. Erroneous Trajectory Estimation}

For the jammer to accurately place a false target at the desired location, it requires knowledge of the seeker position and velocity. Each variable in the time delay equation can be incorrectly estimated by varying amounts. Incorporating these errors into in Eq. (21) gives

$$
\alpha_{\epsilon}=\frac{2}{c}\left[\frac{V_{\epsilon} V\left(x_{\epsilon} x_{0}+\delta_{x}\right)}{\left(R_{\epsilon} R_{0}+\delta_{R}\right)}-\frac{V_{\epsilon} V x_{\epsilon} x_{0}}{R_{\epsilon} R_{0}}\right]
$$

The scalar variables $x_{\epsilon}, R_{\epsilon}$ and $V_{\epsilon}$ are the numerical ratio between the estimated value of the variable and the true value for cross-range, downrange and velocity respectively. 


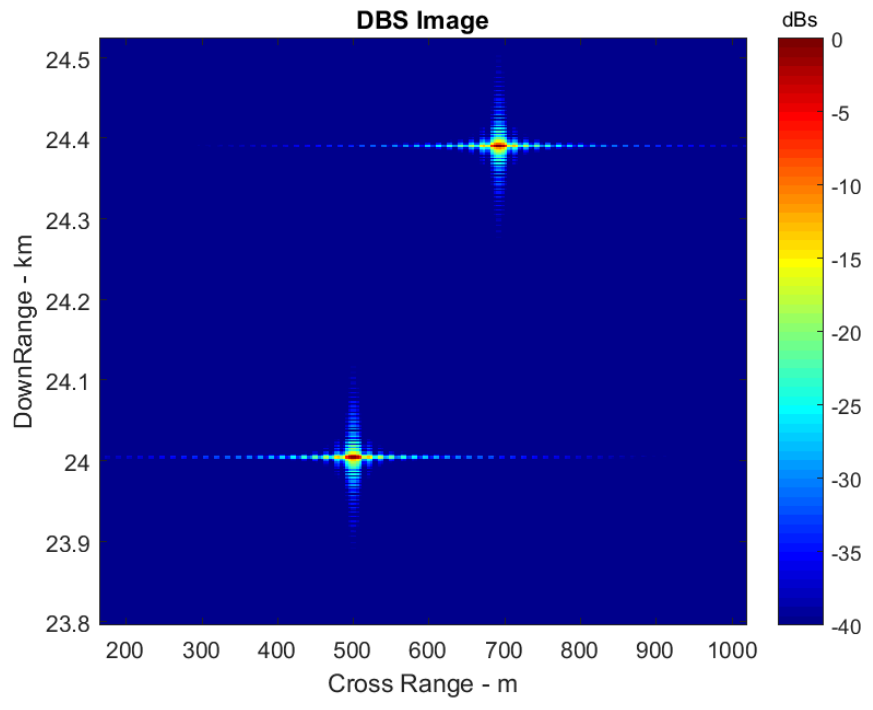

Fig. 3. Induced False Target

For example, if the true velocity of the missile is $300 \mathrm{~ms}^{-1}$ but the jammer estimates it to be $360 \mathrm{~ms}^{-1}$, then $V_{\epsilon}=\frac{V_{j}}{V}=1.2$. Incorporating this into the signal model, the resultant signal would be

$$
\begin{aligned}
s_{4}(t) & =x\left(t-\frac{2 R_{0}}{c}-\tau_{j}\right) e^{i 2 \pi f_{c} \frac{2 \beta t}{c}} \\
& \times e^{-i 2 \pi f_{c}\left(\frac{2 R_{0}}{c}+\tau_{j}\right)} e^{i 2 \pi f_{c} t \frac{2 V_{\epsilon} V}{c}\left[\frac{x_{\epsilon} x_{0}+\delta_{x}}{R_{\epsilon} R_{0}+\delta_{R}}-\frac{x_{\epsilon} x_{0}}{R_{\epsilon} R_{0}}\right]}
\end{aligned}
$$

If there were no errors in the missile trajectory, this would simplify to Eq. (18). By using Eq. (14) to find the instantaneos frequency and therefore cross-range of the false target, it will be seen that the intended Doppler and therefore cross-range position of the target will be shifted, depending on the error.

Using the same scenario as for Figure 3 but with $V_{\epsilon}$ set to 1.2, Figure 4 shows how the position of the false target will be shifted in cross-range. When trying to replicate a complex target with multiple scattering points, this will severely degrade the false target quality.

\section{Practically Modifying a Waveform}

Using the techniques in the previous sections with a Universal Software Radio Peripheral (USRP), a false target was induced into an image at a desired cross-range position of $3 \mathrm{~m}$. With the experiment using the USRP, there was no real movement of the antennas, only a constant delay caused by the separation of the two antennas. The target at $3 \mathrm{~m}$ crossrange was induced by applying an incremental shift to each transmitted pulse by using an assumed missile velocity. This experiment was carried out using a Linear Frequency Modulated (LFM) waveform as per the simulated scenario in Figure 3 . The variables used in the measurement are shown in Table III and the USRP used was a National Instruments NI 2943R. The USRP was controlled using LabVIEW and embedded Matlab software designed the waveforms used. No additional miss distance in downrange was added in this experiment,

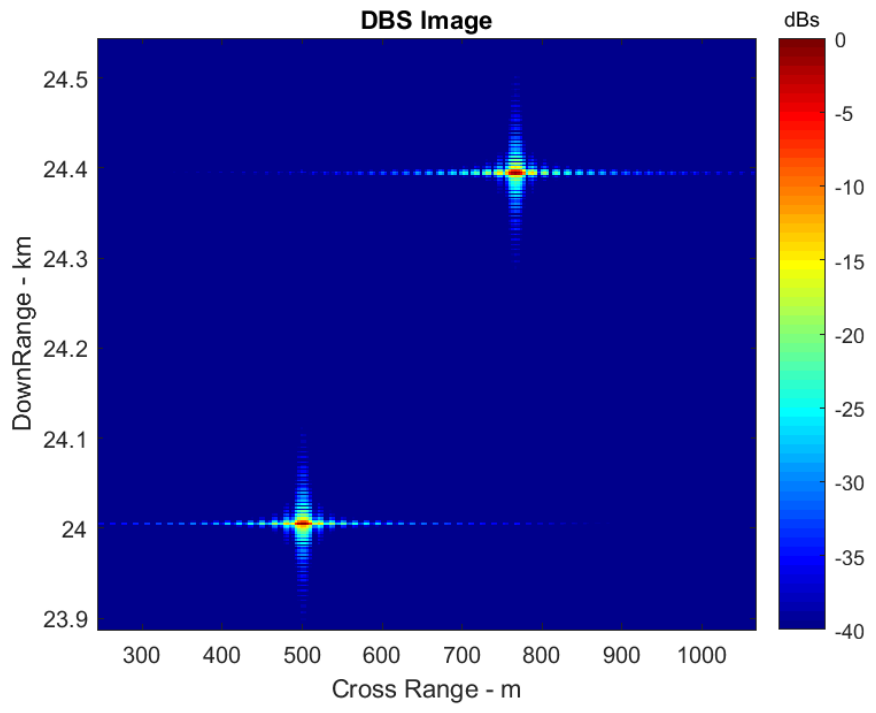

Fig. 4. Shifted False Target with Velocity Magnitude Error

so the quantity $\delta_{R}$ is zero. As there was no real movement of the antennas, the quantity $\beta$ was also zero. Therefore, the transmitted waveform was modified to

$$
x(t)=\sum_{m=0}^{M-1} s_{T}(t-m \mathrm{PRI}) e^{i 2 \pi f_{c} t} e^{i 2 \pi f_{c} \alpha t}
$$

and the received waveform after down-conversion was

$$
\begin{aligned}
s_{R}\left(t_{0}+k \mathrm{PRI}\right) & =\sum_{m=0}^{M-1} s_{T}\left(t_{0}+(k-m) \mathrm{PRI}-\frac{R_{0}}{c}\right) \\
& \times e^{-i 2 \pi f_{c} \frac{R_{0}}{c}} e^{-i 2 \pi f_{c} \alpha \frac{R_{0}}{c}} e^{i 2 \pi f_{c} \alpha t_{0}} e^{i 2 \pi f_{c} \alpha k \mathrm{PRI}}
\end{aligned}
$$

Where for this measurement,

$$
\begin{aligned}
\alpha & =\frac{2 \beta_{J}}{c}=\frac{2 V \delta_{x}}{c R_{0}} \\
s_{T}(t) & =\operatorname{Rect}\left(\frac{t}{\tau}\right) e^{i \pi \gamma t^{2}} \\
\operatorname{Rect}\left(\frac{t}{\tau}\right) & =\left\{\begin{array}{ll}
1, & 0<t<\tau \\
0, & \text { Elsewhere }
\end{array}\right\}
\end{aligned}
$$

TABLE III

USRP VARIABLES

\begin{tabular}{ccc}
\hline \hline Variable & Symbol & Value \\
\hline Carrier Frequency & $f_{c}$ & $4 \mathrm{GHz}$ \\
Bandwidth & $\mathrm{B}$ & $40 \mathrm{MHz}$ \\
Pulse Width & $\tau$ & $10 \mu \mathrm{s}$ \\
Sampling Rate & $F_{s}$ & $80 \mathrm{M}$ \\
Assumed Missile Velocity & $V_{m}$ & $100 \mathrm{~ms}^{-1}$ \\
Pulse Repetition Interval & PRI & $50 \mu \mathrm{s}$ \\
Number of Pulses & $\mathrm{M}$ & 128 \\
Time Delay & $t_{d}$ & $\frac{5}{c}$ \\
\hline
\end{tabular}




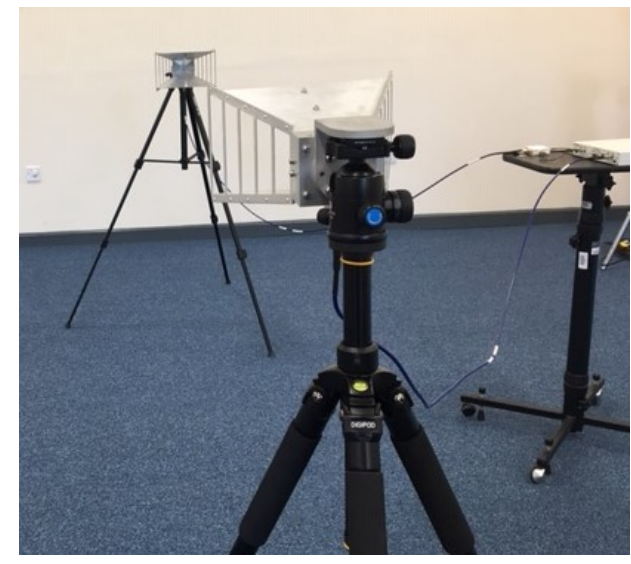

Fig. 5. USRP Set-Up

Figure 5 shows the set-up in the laboratory and Figures 6, 7 and 8 show the results of attempting to induce a false target at $3 \mathrm{~m}$ in cross-range. The ranges and cross-ranges were scaled down for the laboratory, but the method would be the same as shown in the simulation of Figure 3. These figures demonstrate that a false target can be generated at the desired location within a DBS image, providing that the seeker trajectory is known and the carrier frequency and waveform have been estimated correctly.

These measurements were performed for a completely known/assumed trajectory and the next step is to change the experimental set-up to incorporate real movement of the antennas in accordance with the movement of the missile during one CPI.

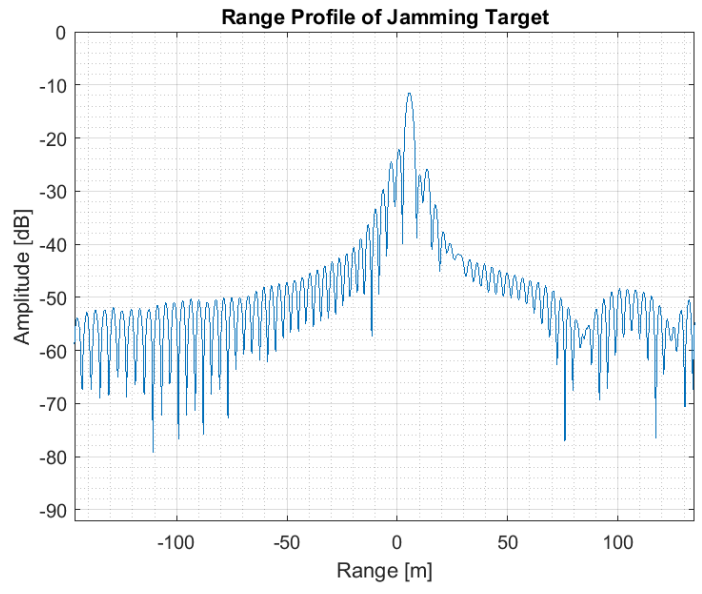

Fig. 6. Range Profile of False Target

\section{CONCLUSION}

A theory of how a jammer can modify a received waveform is presented. The simulations and experiments with the USRP show that waveforms can be sufficiently modified to induce false targets into DBS images at the desired location with relatively inexpensive hardware. With inexpensive hardware,

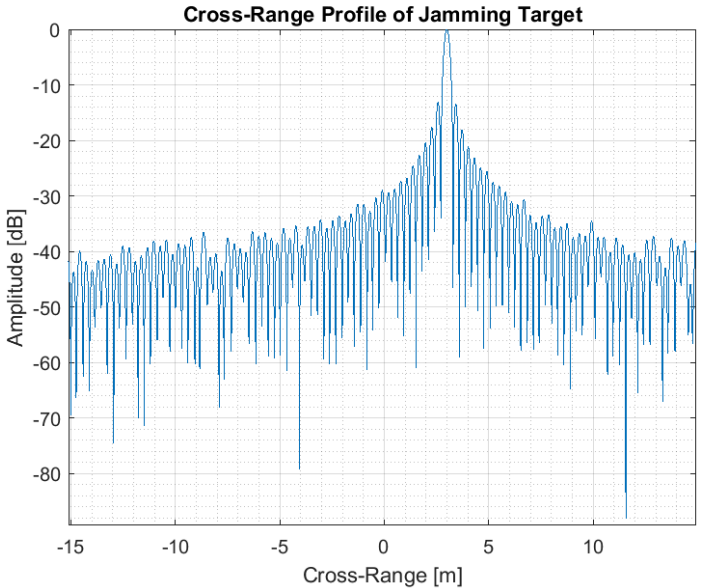

Fig. 7. Cross-Range Profile of False Target

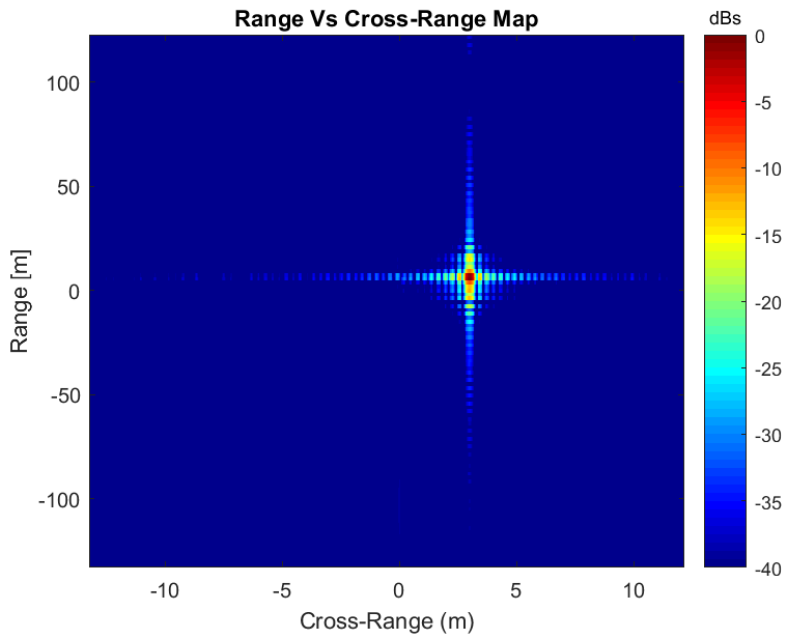

Fig. 8. Induced False Target

the difficulty comes in rapidly creating a digital copy of the received waveform in order to modify it as well as estimating the missile trajectory.

The developed theory incorporates the errors that will be induced into the position and quality of the false target when the missile (or other platform) trajectory is incorrectly estimated. In practice, tracking the missile trajectory is difficult and errors in tracking will degrade the quality of the induced false targets. Erroneous trajectory estimations will shift the position of the target to an undesired location and also cause it to lose focus in the image.

\section{ACKNOWLEDGMENT}

The authors would like to thank The Defence Science and Technology Laboratory (Dstl) and the Engineering and Physical Sciences Research Council (EPSRC) for supporting this research. 


\section{REFERENCES}

[1] Stimson. Introduction to Airborne Radar. Institution of Engineering and Technology, 11998.

[2] Malcolm Rollason, David Salmond, and Michael Evans. Parameter Estimation for Terminal Guidance Using a Doppler Beam Sharpening Radar. In AIAA Guidance, Navigation, and Control Conference and Exhibit, Reston, Virigina, 8 2003. American Institute of Aeronautics and Astronautics.

[3] Asif Farooq and David. J. N. Limebeer. Optimal trajectory tracking for missiles with Doppler Beam Sharpening radars. In Control Conference (ECC), 2007 European. IEEE, 2007.

[4] Jeremy Hodgson and David Lee. Terminal Guidance Using a Doppler Beam Sharpening Radar. In AIAA Guidance, Navigation, and Control Conference and Exhibit, Reston, Virigina, 8 2003. American Institute of Aeronautics and Astronautics.

[5] S.J. Roome. Digital radio frequency memory. Electronics \& Communications Engineering Journal, 2(4):147, 1990.

[6] D A I Da-hai, F Wu, Wang Xue-song, and Xiao Shun-ping. Sar activedecoys jamming based on drfm. In 2007 IET International Conference on Radar Systems, pages 0-3, 2007.

[7] Gareth Frazer, Alessio Balleri, and George Jacob. Simulations of Repeat Jamming against Anti-Ship Missile Seekers Which Use Doppler Beam Sharpening Modes. In 2017 Sensor Signal Processing for Defence Conference (SSPD), pages 1-5. IEEE, 122017. 